\title{
CONSIDERAÇÕES SOBRE O PREPARO DE AMOSTRAS CONTENDO MICROPOLUENTES ESTROGÊNICOS
}

\author{
Marcus Vinicius de Liz \\ Departamento Acadêmico de Química e Biologia, Universidade Tecnológica Federal do Paraná, 80230-901 Curitiba - PR, Brasil \\ Noemi Nagata e Patricio Peralta-Zamora* \\ Departamento de Química, Universidade Federal do Paraná, CP 19081, 81531-990 Curitiba - PR, Brasil
}

Recebido em 8/7/11; aceito em 23/12/11; publicado na web em 23/3/12

\begin{abstract}
CONSIDERATIONS ABOUT THE PREPARATION OF SAMPLES CONTAINING ESTROGENIC MICROPOLLUTANTS. In this work, the materials used in the recovery of estrogens from aqueous matrices by filtration and solid phase extraction were evaluated. The results showed that glass-fiber filters allow a recovery and repeatability compatible with this type of analysis, whereas cellulose esters lead to significant losses of the analytes, mainly due to adsorption processes. On the other hand, the transferring of the sample to the extraction cartridges should be carried out with glass or Teflon tubing, since the adsorption observed with other polymeric materials (eg. silicone, Tygon, polyethylene and PVC) dramatically reduces the recovery and repeatability of the extraction process.
\end{abstract}

Keywords: estrogens; filtration; solid-phase extraction.

\section{INTRODUÇÃO}

A análise de micropoluentes em matrizes complexas provavelmente representa um dos maiores desafios da Química Analítica, principalmente em razão da essencialidade de uma longa sequência de operações preliminares que objetivam a purificação e a pré-concentração das espécies de interesse, mesmo quando se utilizam sistemas de quantificação cada vez mais seletivos e sensíveis (por exemplo, técnicas cromatográficas acopladas a espectrômetros de massas). ${ }^{1,2}$

Embora grande parte destes procedimentos possa ser aplicada com relativa facilidade, a análise de micropoluentes em fase aquosa se apresenta bastante complexa, principalmente em função da possibilidade de perdas da espécie de interesse em cada uma das operações que fazem parte da rotina analítica. Em razão das baixas concentrações usualmente determinadas, o acúmulo de perdas pode redundar em baixa recuperação das espécies de interesse, inviabilizando a análise pretendida.

Em publicação recente, ${ }^{3}$ foram discutidos os principais problemas encontrados na determinação de micropoluentes em amostras de água, particularmente a ocorrência de contaminações que levam a resultados falso-positivos e de perdas associadas à obtenção de resultados falso-negativos. Dentro do contexto dos problemas de contaminação salientam-se contribuições das etapas de coleta, armazenamento e transporte; das condições ambientais do laboratório, da qualidade dos equipamentos e insumos utilizados e, ainda, de contaminações associadas à manipulação por parte do analista, enquanto que em relação às perdas de espécies de interesse salienta-se a capacidade de recuperação das técnicas de extração, as quais costumam ser fortemente influenciadas pela composição da matriz.

Neste trabalho são apresentadas algumas evidências experimentais que demonstram a necessidade de cuidados especiais na seleção de materiais utilizados na manipulação de amostras destinadas à determinação de micropoluentes, tomando como exemplo a extração em fase sólida e a determinação cromatográfica de estrogênios em solução aquosa.

Recentemente foram publicados três trabalhos que discutem o efeito da natureza dos meios filtrantes na recuperação de

\footnotetext{
*e-mail: zamora@ufpr.br
}

estrogênios, ${ }^{4-6}$ o que demonstra a relevância da questão. No primeiro trabalho, ${ }^{4}$ estrona foi utilizada como estrogênio modelo, observando-se significativa adsorção de matérias filtrantes constituídos de nylon, polipropileno, politetrafluoroetileno e acetato de celulose. Em função destes resultados, foi proposta microfibra de vidro como meio filtrante para a remoção de catalisadores em estudos de degradação por fotocatálise heterogênea. No segundo trabalho, ${ }^{5}$ foi estudada a interação entre estrogênios e superfícies poliméricas, avaliando-se o efeito do tipo de polímero, das características do micropoluente e da morfologia das membranas, dentre outros fatores. De maneira geral, foi observado que este tipo de interação é governado por efeitos hidrofóbicos e de solvatação, principalmente envolvendo ligações de hidrogênio e interações dipolo-dipolo, dentre outras. No último trabalho, ${ }^{6}$ foi estudada a adsorção de estradiol em membranas de filtração constituídas de acetato de celulose, particularmente o efeito do $\mathrm{pH}$ e da presença de matéria orgânica. Os resultados demonstram que a presença de coloides orgânicos favorece a adsorção deste tipo de substratos, principalmente em valores de $\mathrm{pH}$ inferiores a 11. Desta forma, o uso deste tipo de membranas exige um ajuste preliminar de $\mathrm{pH}$ em valores superiores a 11 .

\section{PARTE EXPERIMENTAL}

\section{Reagentes e insumos}

17 $\beta$-Estradiol (Sigma, >98\%), Estrona (Sigma, >99\%) e 17 $\alpha-$ -etinilestradiol (Sigma, $>98 \%$ ) foram utilizados em solução aquosa, em concentrações de 10 e $20 \mu \mathrm{g} \mathrm{L}^{-1}$.

$\mathrm{O}$ processo de extração em fase sólida foi realizado em cartuchos Hypersep (500 mg, 3 mL) de sílica C18 (Thermo Electron Corporation).

Os solventes acetonitrila (J.T. Backer), metanol (J.T. Backer) e acetona (Carlo Erba) foram de grau HPLC de pureza. A água ultrapura foi obtida de equipamento Simplicity da Millipore.

Como meios filtrantes foram utilizadas membranas de poliéster de celulose de $0,45 \mu \mathrm{m}(\mathrm{SeS})$, membranas de fibra de vidro de 0,45 $\mu \mathrm{m}(\mathrm{SeS})$ e $0,60 \mu \mathrm{m}(\mathrm{MN})$, e papel filtro faixa azul (CAAL).

Todas as soluções foram preparadas com vidraria analítica previamente calibrada. 


\section{Procedimento de extração em fase sólida}

O processo de extração foi aplicado de acordo com procedimentos descritos na literatura, ${ }^{7,8}$ utilizando-se condicionamento sequencial com acetonitrila $(7 \mathrm{~mL})$, metanol $(5 \mathrm{~mL})$ e água $(5 \mathrm{~mL})$, com vazão de $3 \mathrm{~mL} \mathrm{~min}^{-1}$. Amostras de 100 a $500 \mathrm{~mL}$ foram passadas pela coluna com vazão de $4 \mathrm{~mL} \mathrm{~min}^{-1}$, após o que a coluna foi seca sob fluxo de nitrogênio por $5 \mathrm{~min}$. A eluição foi realizada com quatro porções de $2,5 \mathrm{~mL}$ de acetonitrila, com vazão de aproximadamente $3 \mathrm{~mL} \mathrm{~min} \mathrm{mi}^{-1}$. Finalmente, o solvente foi evaporado até a secura sob fluxo de nitrogênio, o resíduo reconstituído com $1 \mathrm{~mL}$ de metanol e analisado por cromatografia em fase líquida de alta eficiência. Um fluxograma deste procedimento é apresentado na Figura 1.

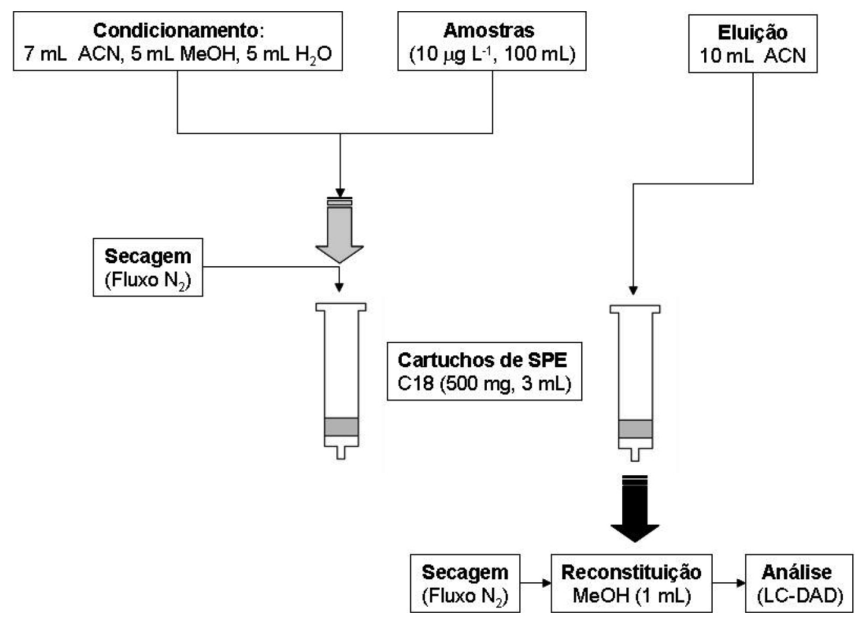

Figura 1. Fluxograma do procedimento de extração em fase sólida

Todos os procedimentos de extração foram realizados em manifold para extração em fase sólida (Varian), equipado com bomba de vácuo e válvula de controle de pressão. A amostra foi passada através dos cartuchos por gotejamento a partir de um funil de adição de vidro (sistema aberto) ou por meio de mangueiras de teflon (e de outros materiais) acopladas ao cartucho (sistema fechado).

Nas condições de análise foram observadas as taxas de recuperação e os desvios apresentados na Tabela 1 .

Tabela 1. Figuras de mérito do método cromatográfico e recuperação média dos estrogênios $\left(20 \mu \mathrm{g} \mathrm{L}^{-1}, \mathrm{n}=5\right)$ no processo de extração em fase sólida

\begin{tabular}{lccc}
\hline \multirow{2}{*}{ Parâmetro } & \multicolumn{3}{c}{ Estrogênio } \\
\cline { 2 - 4 } & E1 & E2 & EE2 \\
\hline Faixa linear de trabalho (mg L & \multicolumn{3}{c}{0,05 a 5,00 } \\
Coeficiente de correlação (r) & 0,9998 & 0,9992 & 0,9970 \\
Limite de detecção (mg L & & & \\
Recuperação média (\%) & 0,009 & 0,018 & 0,035 \\
Desvio padrão (\%) & 97,4 & 99,2 & 98,8 \\
\hline
\end{tabular}

\section{Análise cromatográfica}

As análises cromatográficas foram realizadas em cromatógrafo Varian 920-LC, equipado com detector de arranjo de fotodiodos (197 $\mathrm{nm})$ e coluna C18 microsorb-MV100-5, (250 x 4,6 mm), pré-coluna C8 da mesma marca ( 20 x 4,6 mm), utilizando-se fase móvel constituída de acetonitrila:água (50:50, v:v) com vazão de $0,8 \mathrm{~mL} \mathrm{~min}{ }^{-1}$.

A curva analítica foi preparada a partir de 6 soluções padrão de calibração, contendo a mistura dos 3 estrogênios em fase móvel, cobrindo-se a faixa de 0,03 a $5,0 \mathrm{mg} \mathrm{L}^{-1}$ de cada estrogênio. Com base nestas curvas foram calculadas as figuras de mérito apresentadas na Tabela 1.

\section{RESULTADOS E DISCUSSÃO}

Inicialmente foi avaliado o efeito de diversos materiais filtrantes na recuperação dos estrogênios, a partir de $100 \mathrm{~mL}$ de solução a 20 $\mu \mathrm{g} \mathrm{L} \mathrm{L}^{-1}$, obtendo-se os resultados apresentados na Figura 2. Estes resultados demonstram a relevância, não apenas do material de filtração, mas também do suporte utilizado. De maneira geral, o uso de vidro sinterizado se mostrou inconveniente para o suporte de membranas de éster de celulose e fibra de vidro, enquanto que o funil de Buchner, feito de porcelana, permitiu recuperações próximas à do sistema controle, para ambas as membranas. Por sua vez, o uso de papel quantitativo denso (faixa azul) apresentou os piores resultados de recuperação, principalmente para estrona, o que pode ser atribuído à existência de interação por pontes de hidrogênio entre os estrogênios e a celulose. ${ }^{9}$

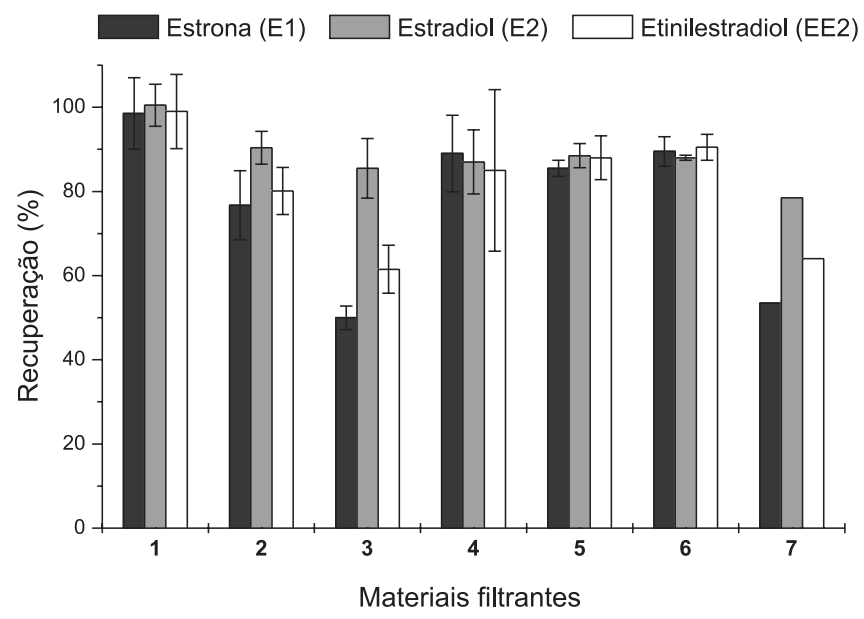

Figura 2. Recuperação de hormônios para diferentes materiais filtrantes testados a partir de $100 \mathrm{~mL}$ de solução contendo $20 \mu \mathrm{g} \mathrm{L} \mathrm{L}^{-1}$ de cada estrogênio ( $n=3$, 1: sem filtração, 2: membrana de poliéster de 0,45 $\mu \mathrm{m}$ em funil de Gooch, 3: fibra de vidro de 0,45 $\mu$ m em funil de Gooch, 4: membrana de poliéster de 0,45 $\mu \mathrm{m}$ em funil de Büchner, 5: fibra de vidro de 0,45 $\mu \mathrm{m}$ em funil de Büchner, 6: fibra de vidro de 0,60 $\mu \mathrm{m}$ em funil de Büchner, 7: papel de filtro faixa azul em funil de Büchner)

Estes resultados também são coerentes com alguns relatos da literatura, que indicam adsorção de estrona da ordem de $20 \%$ em filtros de acetato de celulose e da ordem de $2 \%$ em filtros de fibra de vidro. ${ }^{4}$ Outros trabalhos demonstram perdas de até $50 \%$ no teor nominal de $17 \beta$-estradiol ( 50 a $150 \mathrm{ng} \mathrm{L}^{-1}$ ), em processos de filtração envolvendo membranas de acetato de celulose. ${ }^{6}$

Posteriormente, objetivando-se melhorar o sistema de transferência da amostra para o cartucho de extração, a alimentação manual (funil de adição) foi substituída por um sistema semiautomático fundamentado no uso de manifold de extração, bomba de vácuo e mangueiras de condução. Este sistema permitiu a passagem simultânea de 5 amostras de $100 \mathrm{~mL}$, contendo os estrogênios em concentração de $20 \mu \mathrm{g} \mathrm{L} \mathrm{L}^{-1}$.

Os materiais avaliados para condução das amostras até o cartucho foram Teflon, silicone, tygon, polietileno e PVC, que permitiram a obtenção das taxas de recuperação apresentadas na Figura 3. De acordo com estes resultados, grande parte dos materiais leva à obtenção de baixas taxas de recuperação, assim como de desvios de grande magnitude, principalmente em função de adsorção dos substratos em estudo. 


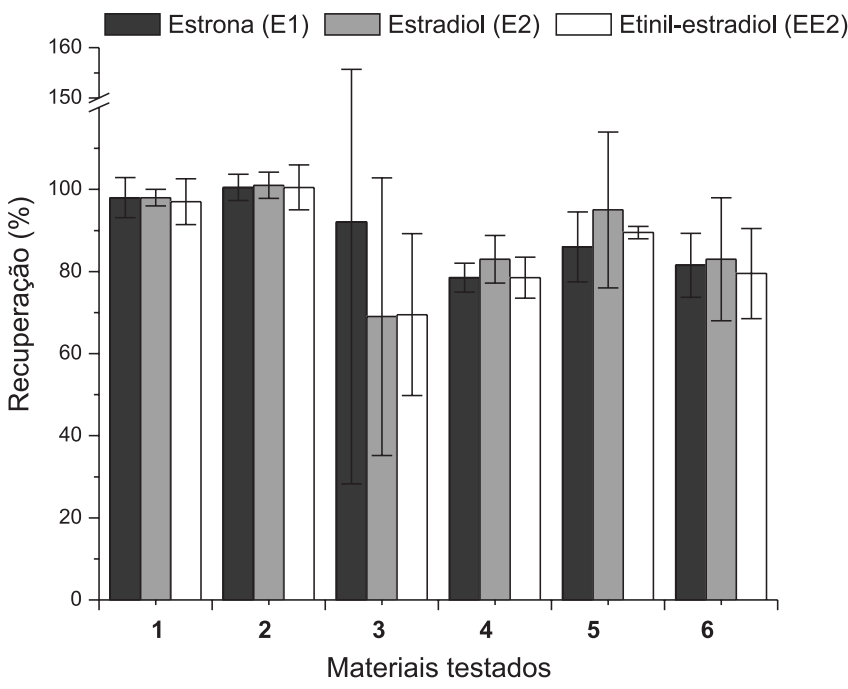

Figura 3. Recuperação de hormônios para diferentes materiais utilizados para condução das amostras até o cartucho de extração $(n=3,1$ : vidro, 2 : Teflon, 3: silicone, 4: tygon, 5: polietileno, 6: PVC)

A este respeito, alguns trabalhos alertam que os valores de $\mathrm{K}_{\mathrm{ow}}$ dos estrógenos $(2,6$ a 4,0) indicam uma tendência de interação com substâncias apolares, ${ }^{9}$ embora outras propriedades funcionais dos compostos também devam ser levadas em consideração. Em geral, estima-se que os processos referidos de sorção podem ser devidos à formação de ligações de hidrogênio, as quais são favorecidas pelo caráter próton-doador do grupo fenólico dos estrogênios. ${ }^{4}$ Adicionalmente, sugere-se que os grupos fenólicos, ricos em elétrons $\pi$, podem propiciar interações do tipo $\pi$ - $\pi$ com grupos fenil deficientes em elétrons dos materiais poliméricos. ${ }^{5}$

Dentre os materiais testados, os únicos que poderiam ser utilizados para transferência das soluções de Estrona, Estradiol e Etinilestradiol do frasco de amostra para o cartucho de SPE são vidro e teflon, os quais permitiram recuperações médias entre 97 e $102 \%$ de todos os estrogênios em estudo, com desvios padrão relativos da ordem de 5\%. Estes resultados são coerentes com os antecedentes relatados na literatura, ${ }^{4}$ que sugerem o uso de teflon, em função da baixa adsorção apresentada em relação à estrona.

É importante salientar que a filtração de amostras aquosas contendo matéria orgânica ou outras substâncias coloidais acrescenta problemas operacionais adicionais, relacionados com o entupimento dos poros da fase de membrana (clogging) e com a formação de depósitos que praticamente agem como uma segunda membrana (fouling). Estudos recentes demonstram que o fenômeno de fouling associado à presença de ácidos húmicos e polissacarídeos aumenta significativamente a retenção de estradiol em processos de ultrafiltração. ${ }^{10}$

Este tipo de efeito é particularmente importante na filtração de amostras mais complexas, como águas naturais e esgoto. Neste caso, fenômenos de clogging e fouling são bastante comuns, ${ }^{11}$ o que limita o volume de amostra, aumenta o tempo de filtração e diminui a capacidade de recuperação de certos micropoluentes. ${ }^{12}$

Neste estudo, amostras de esgoto bruto e tratado foram analisadas em relação ao teor de estrogênios, utilizando-se a rotina de extração em fase sólida seguida de quantificação cromatográfica. Em primeiro lugar, é importante salientar que a presença de materiais coloidais nas amostras de esgoto impediu o uso de amostras de $500 \mathrm{~mL}$ no processo de extração, principalmente em função do entupimento dos poros do material sorbente reduzir drasticamente a vazão de passagem das amostras. Trata-se de um antecedente relevante, não apenas em razão do significativo aumento no tempo de análise, mas especialmente em razão de perdas associadas ao fenômeno de fouling.

Finamente, é pertinente reiterar que são inúmeros os fatores que contribuem, não apenas com a perda das espécies de interesse, mas também com a contaminação das amostras, característica esta última que pode levar à geração de resultados falso-positivos. ${ }^{3}$ Dentro deste contexto, deve ser dado destaque a processos de contaminação envolvendo ftalatos, a partir de fontes de contaminação que podem ser evidentes (por exemplo, mangueiras e vasilhames plásticos) ${ }^{13} \mathrm{ou}$ não tão evidentes (como ar atmosférico, solventes, materiais de vidro e septos de borracha, dentre outros). ${ }^{14}$

\section{CONCLUSÕES}

De maneira geral, a análise de traços em matrizes ambientais se mostra bastante complexa, principalmente em função da necessidade de rotinas que envolvem múltiplas etapas auxiliares. Na análise de estrogênios, por exemplo, devem ser realizadas com cautela as operações de filtração, extração e condução de amostras, em função das inúmeras possibilidades de perda por adsorção dos analitos nos materiais utilizados.

Neste caso em particular, o uso de materiais filtrantes à base de ésteres de celulose se mostrou inconveniente, principalmente em função da adsorção de estrogênios implicar em baixas taxas de recuperação $(<90 \%)$. Contrariamente, o uso de filtros constituídos de fibra de vidro permite recuperações médias da ordem de $95 \%$, o que se mostra adequado para este tipo de análise.

Dos materiais utilizados para condução das amostras até os cartuchos de extração, apenas vidro e Teflon permitiram taxas de recuperação compatíveis com os objetivos do estudo. Outros materiais (por exemplo, silicone, tygon, polietileno e PVC), além de proporcionarem baixas taxas de recuperação, introduzem flutuações que comprometem a repetibilidade do processo.

\section{REFERÊNCIAS}

1. Pedrouzo, M.; Borrull, F.; Pocurull, E.; Marcé, R. M.; Talanta 2009, 78, 1327.

2. Yan, W.; Li, Y.; Zhao, L.; Lin, J.-M.; J. Chromatogr., A 2009, 1216, 7539.

3. Capdeville, M. J.; Budzinski, H.; Trends Anal. Chem. 2011, 30, 586.

4. Han, H.; Qiu, W.; Gao, W.; Chem. Eng J. 2010, 165, 819.

5. Schäfer, A. I.; Akanyeti, I.; Semião, A. J. C.; Adv. Colloid Interface Sci. 2011, 164, 100.

6. Neale, P. A.; Pronk, W.; Schäfer, A. I.; Environ. Eng. Sci. 2009, 26, 1157.

7. Girotto, G.; Nogueira, K. L.; Moura, J. A.; Souza, J. B.; Giacometti, L. L..; Marchi, M. R.R.; Tognolli, J. O.; Eclet. Quím. 2007, 32, 61.

8. López de Alda, M. J.; Barceló, D.; J. Chromatogr., A 2001, 938, 145.

9. Jones, O. A. H.; Voulvoulis, N.; Lester, J. N.; Crit. Rev. Environ. Sci. Technol. 2005, 35, 401.

10. Jermann, D.; Pronk, W.; Boller, M.; Schäfer, A. I.; J. Membr. Sci. 2009, $329,75$.

11. Mocé-Llivina, L.; Cofre, J.; Muniesa, M.; J. Virol. Methods 2003, 109, 99.

12. Baker, D. R.; Kasprzyk-Hordern, B.; J. Chromatogr., A 2011, 1218, 8036.

13. Jenke, D. R.; Story, J.; Lalani, R.; Int. J. Pharm. 2006, 315, 75.

14. Fankhauser-Noti, A.; Grob, K.; Anal. Chim. Acta 2007, 582, 353. 